Mit Rücksicht auf diese umfangreiche Tätigkeit des Hofrates Dr. Melichar können wir wohl den Wunsch aussprechen, es möchte ihm gestattet sein, noch lange Jahre im Interesse der Wissenschaft und zum Wohle der leidenden Menschheit zu wirken!

\title{
Die Typen der Pilzmücken der von Roserschen Sammlung in Stuttgart.
}

Von Karl Landrock, Brünn.

Jedem Dipterologen, der ernstlich seinen Studien obliegt und zur Bestimmung seiner Tiere womöglich alle vorhandenen dipterologischen Arbeiten zu verwenden sucht, werden gewif auch die von R o serschen Beschreibungen im Korrespondenzblatte des k. württemberg. landwirtsch. Vereines, Jahrg. 1840, Band I, Heft 1, bekannt sein. Aber schon bei der Beschaffung des genannten Werkes stößt man meist auf große Schwierigkeiten, denn dasselbe ist im Buchhandel überhaupt nicht zu erlangen und fehlt vielfach auch in sonst umfangreichen Büchereien. Hat man es endlich durch das freundliche Entgegenkommen irgend eines Herrn Bibliothekars erlangt, so wird man von den Beschreibungen ganz enttäuscht, denn sie sind alle so kurz und knapp gehalten und entsprechen dem heutigen Stande der Wissenschaft so wenig, daß ein Erkennen einer Roserschen Art sehr schwer, in den meisten Fällen überhaupt unmöglich ist. Da gab es nur e in en Weg, um hier Klarheit zu schaffen, die Revision der Roserschen Typen.

Dieser Arbeit hat sich in dankenswerter Weise schon im Jahre 1902 Herr Th. Becker aus Liegnitz unterzogen, einen Teil der Roserschen Sammlung, die Diptera cyclorrhapha schizophora (Muscaria holometopa), revidiert und das Ergebnis seiner Untersuchungen in dem Jahresheft des Vereines für vaterländische Naturkunde in Württemberg, 1903, p. 52-66, niedergelegt. Leider umfaft diese vortreffliche Arbeit nur die oben genannte Dipterengruppe und es blieben noch immer viele Typen der Roserschen Sammlung übrig, die einer weiteren Bearbeitung harrten.

Um auch bezüglich der Familie der Nycetophiliden Klarheit $\mathrm{zu}$ bekommen, habe ich mich in einem Schreiben an den Verein für vaterländische Naturkunde in Württemberg, in dessen Besitz sich die Sammlung befindet, mit der Bitte gewendet, mir die Roserschen 
Typen dieser Familie zur Revision zu überlassen. $\mathrm{Zu}$ meiner Befriedigung hat mir Herr Oberstudienrat Dr. Lampert in zuvorkommendster Weise diese Typen zugesichert, anerkennend den Umstand, daß dieselben einer Revision benötigen, und der Kustos Herr Dr. E. Lindner hat mir anfangs Jänner nicht bloß die Typen der Sammlung zugeschickt, sondern in liebenswürdiger Weise auch noch andere Pilzmücken der von Roserschen Sammlung der Sendung beigesteckt. Genannten Herren sei hierorts nochmals mein bester Dank zum Ausdrucke gebracht.

Nachstehend das Ergebnis meiner Untersuchungen.

\section{Familie: Mycetophilidae.}

1. Bolitophila bimaculata v. Ros., p. 51.

Unter diesem Namen fand ich vier Mücken vor. Zwei davon ( $(q)$ sind, wie ich schon früher vermutet habe (vergl. B. E. Z., 1912, p. 35), Bolitophila bimaculata Zetterstedt. Das dritte Tier ist eine Rhipidia maculata Mg. O, das vierte ist ein Rudiment (ohne Beine und Hinterleib, mit zerknitterten Flügeln) einer Mycomya (Sciophila), nach der über dem Zellchen abgebrochenen Subcosta $\left(\mathrm{sc}_{1}\right)$ zu schließen, Mycomya incisurata Zett.

2. Macrocera fusciventris v. Ros., p. 51.

Eine Type dieser Art ist in der Sammlung nicht vorhanden. 3. Platyura bifasciata v. Ros., p. 51.

1 万o ist Leia (Glaphyroptera Winn., Neoglaphyroptera O. S.) subfasciata Mg.

4. Platyura fuscescens v. Ros., p. 51.

Von dieser Art fand ich vier Stück vor. 1 ist gut erhalten, $1 \sigma^{7}$ fehlen Kopf und Flügel, den zwei anderen Stücken der Hinterleib. Drei Stücke davon sind bestimmt Platyura nemoralis Mg., das vierte rudimentäre Stück hat einen gelben Thoraxrücken, gleicht aber im Flügelgeäder und in der Flügelzeichnung ganz den anderen. Das Hypopygium des Männchens stimmt genau mit der Zeichnung Edwards in Trans. of ent. soc. Lond., 1913, t. 13, fig. 20, t. 14, fig. 21, überein.

5. Platyura lata v. Ros., p. 51.

Hier fanden sich drei Stücke vor; 1 , bis auf die Beine, gut erhalten, 1 † ohne Fühler und mit stark beschädigten Flügeln, von dem dritten haftete an der Nadel allerdings nur ein Flügel. Alle drei sind Platyura flava Macq. 
6. Sciophila bimaculata v. Ros., p. 51.

An der Nadel befindet sich ein Hinterbein und ein Flügel, alles andere fehlt, dennoch glaube ich die Art deuten zu können. Es ist Neoempheria (Empheria Winn.) formosa Winn. Im Flügelgeäder stimmt diese Art ganz mit der Winnertzschen Beschreibung überein, doch steht $\mathrm{sc}_{2}$ etwa eine Aderbreite vor der Basis von rs. Zwischen $r_{4}+5$ und $m_{1}$ ist eine ziemlich tiefe Flügelfalte, die, einer blassen Längsader ähnlich, gegen die Flügelspitze zieht, ein Stück vor derselben jedoch abbricht. W innertz erwähnt von dieser Flügelfalte, die übrigens (aber schwächer) auch bei anderen Neoempheria-Arten vorkommt, kein Wort. Die Flügelzeichnung stimmt ganz mit der Winnertzschen Beschreibung, doch ist die Mittelbinde im unteren Teile etwas verblaßt.

In der Sammlung steckt übrigens noch ein zweites Stück dieser Art, und zwar bei Leia bifasciata, doch hat dasselbe nur einen Flügel und der ist im Spitzenteile und am Hinterrande abgerissen, so daf der Aderverlauf und die Flügelzeichnung nicht vollständig ersichtlich sind. Die Färbung des Rückenschildes und des Hinterleibes stimmen mit der Winnertzschen Beschreibung, doch dehnt sich die weibgelbe Färbung des Bauches auf dem vierten Ringe etwas auf die Seiten aus. Die Oberseite dieses Ringes ist jedoch schwarzbraun.

7. Sciophila flavipennis v. Ros., p. ว́1.

Ist Sciophila (Lasiosoma Winn.) lutea Macq. 1 q gut erhalten. Das zweite Stuick ist ganz zerfressen, der Kopf, der größte 'T'eil des Mittelleibes und die Beine fehlen. Die Flügel sind bei diesem Stücke etwas breiter, $\mathrm{Sc}_{2}$ steht vor dem Zellchen und ax ist etwas derber als bei dem anderen Exemplare.

8. Lèia bivittata v. Ros., p. 51.

Zwei Exemplare sind gut erhalten, das dritte mit ganz zerfressenem Thorax, ohne Hinterleib und ganz zerknitterten Flügeln. Die Art ist identisch mit Leia (Neoglaphyroptera O. S., Glaphyroptera Winn.) picta $\mathrm{Mg}$.

9. Leia bifasciata v. Ros., p. 51.

Ein gut erhaltenes on ist Mycetophila cingulum Meig., ein zweites Stiick ist, wie schon erwähnt (vergl. 6), Empheria formosa Winn.

10. Leia fuscipes v. Ros., p. 51.

Vier $q$ o und ein oc sind Docosia valida Winn. im Sinne Winnertz, Verh. z. b. Ges., 1863. Ich habe D. valida Winn. 
(vergl. Zeitschr. d. mähr. Land.-Mus., 1916) in mehrere Arten zerlegt, die einander im Habitus gleichen, aber durch das Hypopygium verschieden sind. Nach dieser Auffassung stellt das on Docosia pseudovalida Land. dar. Ein sechstes Stück ist Azana anomala Staeg.

11. Leia humulata v. Ros., p. 51.

2 우 und $1 \sigma^{\top}$, gut erhalten, sind Leia subfasciata Mg. (vergl. 3).

12. Leia apicalis v. Ros., p. 51.

$1 \delta$ ist ein schönes, gut erhaltenes Stück der seltenen Art Leptomorphus Walkeri Curt.

13. Mycetophila flaviventris v. Ros., p. 51.

1 ठ", gut erhalten, ist Leia (Neoglaphyroptera O. S., Glaphyroptera Winn.) bimaculata Meig.

14. Mycetophila crassicornis v. Ros., p. 51.

Eine Type dieser Art fehlt in der Sammlung.

Aus Vorstehendem ergibt sich somit folgende Synonymie:

Macrocera fusciventris v. Ros., läßt sich nicht deuten; keine Typen vorhanden.

Mycetophila crassicornis v. Ros., läßt sich nicht deuten; keine Typen vorhanden.

Bolitophila bimaculata Zett. $=$ Bolitophila bimaculata v. Ros.

Platyura nemoralis Meig. = Platyura fuscescens v. Ros.

Platyura flava Nacq. = Platyura lata v. Ros.

Sciophila lutea Nacq. = Sciophila flavipennis v. Ros.

Leptomorphus Walkeri Curt. = Leia apicalis v. Ros.

Leia subfasciata Meig. = Platyura bifasciata v. Ros.

= Leia Tumulata v. Ros.

Leia picta Mg. = Leia bivittata v. Ros.

Leia bimaculata Mg. = Mycetophila flavicentris v. Ros.

Mycetophila cingulum Mg. = Leia bifasciata v. Ros.

Neoempheria bimaculata v. Ros. = Neoempheria formosa Winn.

Docosia fuscipes v. Ros. = Docosia valida Winn. p. p.

$=$ Docosia pseudovalida Land. 


\section{$2 \mathrm{BHL}$ Biodiversity Heritage Library}

Landrock, Karl. 1917. "Die Typen der Pilzmücken der von ROSERschen

Sammlung in Stuttgart." Wiener entomologische Zeitung 36, 36-39.

https://doi.org/10.5962/bhl.part.12922.

View This Item Online: $\underline{\text { https://www.biodiversitylibrary.org/item/42783 }}$

DOI: https://doi.org/10.5962/bhl.part.12922

Permalink: https://www.biodiversitylibrary.org/partpdf/12922

\section{Holding Institution}

Smithsonian Libraries

\section{Sponsored by}

Smithsonian

\section{Copyright \& Reuse}

Copyright Status: NOT_IN_COPYRIGHT

This document was created from content at the Biodiversity Heritage Library, the world's largest open access digital library for biodiversity literature and archives. Visit BHL at https://www.biodiversitylibrary.org. 